\title{
Health Education "Hokenshido" Program Reduced Metabolic Syndrome in the Amagasaki Visceral Fat Study. Three-Year Follow-up Study of 3,174 Japanese Employees
}

\author{
Miwa Ryo ${ }^{1}$, Tadashi Nakamura ${ }^{1}$, Tohru Funahashi ${ }^{1}$, Midori Noguchi ${ }^{2}$, Ken Kishida ${ }^{1}$, \\ Yukiyoshi Okauchi ${ }^{1}$, Hitoshi Nishizawa ${ }^{1}$, Tomoko Ogawa ${ }^{2}$, Sumi Kojima ${ }^{2}$, Tetsuya Ohira ${ }^{3}$, \\ Kohei Okita ${ }^{1}$, Hiromi Iwahashi ${ }^{1}$, Akihisa Imagawa ${ }^{1}$, \\ Yuji Matsuzawa ${ }^{4}$ and Iichiro Shimomura ${ }^{1}$
}

\begin{abstract}
Objective The aim of this study was to evaluate the effects of health checkup and the health education "Hokenshido" program based on the concept that visceral fat accumulation causes metabolic syndrome (MetS), leading to cardiovascular disease (CVD).

Methods and Subjects Based on the Japanese definition of metabolic syndrome, in the annual health checkup for general subjects, the measurement of waist circumference and use of "Where am I?" chart on the way to develop atherosclerosis were introduced. The study group comprised 3,174 Japanese employees [2,440 males ( $46 \pm 11$ years, mean \pm SD), 734 females $(43 \pm 10$ years)], who underwent annual health checkup in 2003, 2004, and 2005. The medical staff provided "Hokenshido" for subjects assessed as having MetS and/or at high risk for CVD.

Results The prevalence of the MetS in 2003, 2004 and 2005 decreased in males $(20.8 \%, 17.2 \%, 14.4 \%$, p $<$ 0.001 ) and females $(3.0 \%, 2.2 \%, 1.9 \%, \mathrm{p}=0.359)$, respectively. Among subjects with MetS at baseline, the number of subjects with MetS significantly decreased in males $(508,287,247, \mathrm{p}<0.0001)$ and females $(22,8$, $6, \mathrm{p}<0.0001)$, respectively. Mean waist loss was $1.6 \mathrm{~cm}$ in males $(<0.0001)$ and $1.5 \mathrm{~cm}$ in females $(<0.001)$. Among subjects with metabolic syndrome at baseline, the mean waist loss was $2.5 \mathrm{~cm}$ in males $(<0.0001)$ and $3.9 \mathrm{~cm}$ in females $(<0.05)$. Fatal atherosclerotic vascular events were not recorded in this study period. Conclusion Health check-up and the "Hokenshido" program reduced the prevalence of the MetS, which might lead to prevention of CVD.
\end{abstract}

Key words: metabolic syndrome, health checkup, health guidance, Hokenshido, visceral fat accumulation, cardiovascular disease

(Intern Med 50: 1643-1648, 2011)

(DOI: 10.2169/internalmedicine.50.5039)

\section{Introduction}

The metabolic syndrome is a risk factor for atherosclerotic cardiovascular diseases (CVD) (1). Visceral fat accumulation caused by overnutrition and physical inactivity is closely related to glucose intolerance, dyslipidemia, hypertension, and CVD (2-4). Once a diagnosis of the metabolic syndrome is established, management of the condition, lifestyle change as the primary intervention and assessment of cardiovascular risk factors should be adequately conducted to reduce the risk of CVD (5-7).

\footnotetext{
${ }^{1}$ Department of Metabolic Medicine, Graduate School of Medicine, Osaka University, Japan, ${ }^{2}$ Amagasaki City Office, Environment \& Civic Affairs Bureau Health Support Promotion Section, Japan, ${ }^{3}$ Department of Social and Environmental Medicine, Graduate School of Medicine, Osaka University, Japan and ${ }^{4}$ Sumitomo Hospital, Japan

Received for publication December 21, 2010; Accepted for publication April 4, 2011

Correspondence to Dr. Miwa Ryo, ryomw@gaia.eonet.ne.jp
} 
Table 1. Clinical Characteristics of the Subjects at Baseline

\begin{tabular}{lcc}
\hline Variables & Male $(\mathrm{n}=2440)$ & Female $(\mathrm{n}=734)$ \\
\hline Age, years & $45.5 \pm 10.6$ & $43.0 \pm 9.7$ \\
Body weight, $\mathrm{kg}$ & $69.4 \pm 9.8$ & $54.6 \pm 8.5$ \\
Body mass index, $\mathrm{kg} / \mathrm{m}^{2}$ & $24.3 \pm 3.1$ & $22.3 \pm 3.5$ \\
Waist circumference, cm & $84.9 \pm 8.3$ & $78.2 \pm 10.0$ \\
Triglyceride, $\mathrm{mg} / \mathrm{dL}$ & $174.1 \pm 123.1$ & $105.3 \pm 75.1$ \\
HDL cholesterol, mg/dL & $53.7 \pm 14.7$ & $65.4 \pm 15.4$ \\
LDL cholesterol, mg/dL & $115.4 \pm 29.7$ & $111.7 \pm 28.7$ \\
Systolic blood pressure, mm Hg & $130.3 \pm 15.7$ & $119.2 \pm 15.6$ \\
Diastolic blood pressure, mm Hg & $80.1 \pm 11.8$ & $71.9 \pm 11.9$ \\
Plasma glucose, mg/dL & $105.3 \pm 33.4$ & $98.3 \pm 24.7$ \\
\hline
\end{tabular}

Data are means \pm SD. HDL, high-density lipoprotein; LDL, low-density lipoprotein.

In Amagasaki City Office, 7 fatal atherosclerotic vascular events were recorded from year 1995 to 2002 in all $-4,000$ employees. In addition to the health costs, the insurance costs had also been increasing. Therefore, prevention of CVD was an important and urgent task for the city and employer. To this end, measurement of waist circumference in annual health checkup commenced in year 2003 in these employees, based on the concept that visceral fat accumulation causes the metabolic syndrome. According to the results of the health checkup, a health education "Hokenshido" program, using "Where am I?" chart on the way to develop atherosclerosis, was applied by the medical staff to prevent further development of lifestyle-related diseases and CVD for each subject. We reported previously that the decrease in visceral fat within one year correlated with the decrease in the number of metabolic risk factors (raised blood pressure, dyslipidemia and glucose intolerance) and increase in serum levels of adiponectin (8-17).

The aim of this study was to evaluate the effect of this whole program on the incidence of metabolic syndrome for each year and for each generation of males and females.

\section{Materials and Methods}

\section{Participants}

This urban area study group comprised 3,174 Japanese [2,440 males $(45.9 \pm 10.6$ years, mean \pm SD), 734 females (43.0 \pm 9.7 years)] who were employees of the Amagasaki City Office, Hyogo, Japan and had completed the Government-funded annual health checkup every year from 2003 to 2005. The clinical characteristics of the study participants at baseline in year 2003 are shown in Table 1. Of the entire group, $118(3.7 \%), 337$ (10.6\%), and $115(3.6 \%)$ individuals were under treatment for dyslipidemia, hypertension, or diabetes, respectively, at baseline.

All participants gave full informed consent to participate in the study and ethical approval was obtained from committee on the Ethics of Human Research of Osaka University. This trial is registered with number UMIN 000002391 (the Amagasaki Visceral Fat Study).

\section{Anthropometry and laboratory measurements}

Height and weight were measured in the standing position. Body mass index was calculated as weight $(\mathrm{kg})$ divided by the square of height in meters $\left(\mathrm{m}^{2}\right)$. Waist circumference at the umbilical level was measured in $\mathrm{cm}$ with a nonstretchable tape in the late exhalation phase at standing position (18). Systolic and diastolic blood pressure values were measured in the sitting position. Blood was withdrawn fasting or postprandial condition. Biochemical variables were measured with a conventional automated analyzer.

\section{Assessment of risk factors}

We defined the metabolic syndrome according to the guidelines for the diagnosis in Japan (19). Abdominal obesity, waist circumference equal to or greater than $85 \mathrm{~cm}$ in men or greater than $90 \mathrm{~cm}$ in women plus the presence of at least two of the following abnormalities: 1) dyslipidemia; a serum fast triglyceride level over $150 \mathrm{mg} / \mathrm{dL}$ and/or a serum high-density lipoprotein (HDL) cholesterol level less than 40 $\mathrm{mg} / \mathrm{dL}, 2)$ hypertension; systolic blood pressure over 130 $\mathrm{mmHg}$ and/or diastolic blood pressure over $85 \mathrm{mmHg}$ and 3) high glucose; serum fast glucose level over $110 \mathrm{mg} / \mathrm{dL}$. Subjects who received specific treatment(s) for each of the above metabolic risk factors were considered positive for that factor. It means that, those who had a risk factor without treatment and those who were on treatment were also included as study subjects. In the case that blood samples were not obtained after $>8$-hour fasting, we modified 1) to 1)' dyslipidemia; postprandial triglyceride level over 200 $\mathrm{mg} / \mathrm{dL}(20,21)$ and/or a serum high-density lipoprotein (HDL) cholesterol level less than $40 \mathrm{mg} / \mathrm{dL}, 3$ ) to 3)' as high glucose; postprandial serum glucose level over $140 \mathrm{mg} /$ dL (22).

\section{Detailed examination}

Oral glucose tolerance test, bicycle ergometer stress test, and carotid artery echography were performed in those subjects with risk factor(s) based on the recommendation of the team physician. 
"Where am I ?" chart

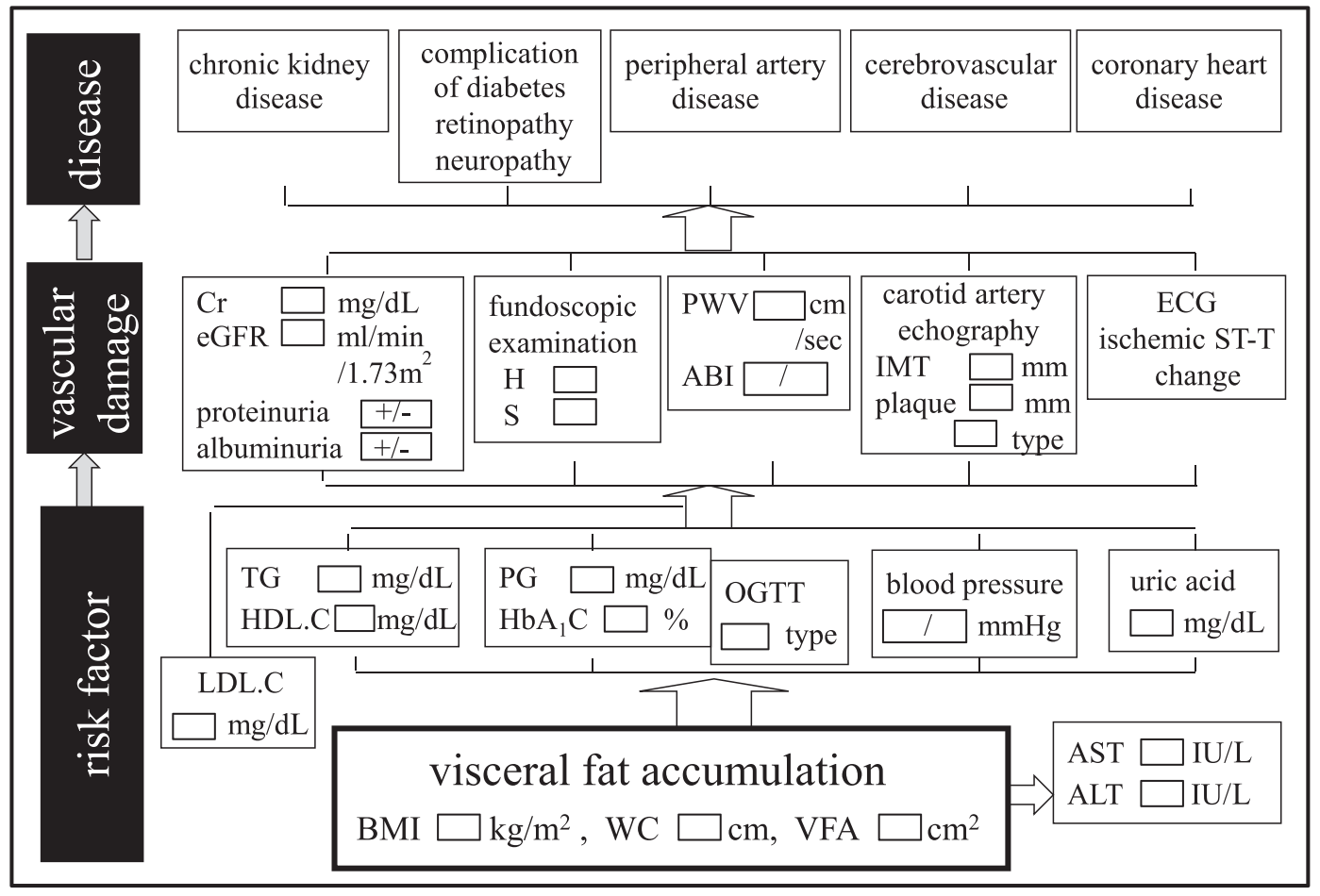

Figure 1. The "Where am I?" chart. All results were transferred into this chart for the individual subject. The metabolic risk factors of vascular damage were displayed at the bottom part of the chart. The results of detailed examination to estimate the current condition of vascular damage were set at the middle of the chart. The status of presence or absence of diseases such as cardiovascular diseases (CVD) was displayed at the top of the chart. BMI: body mass index, WC: waist circumference, VFA: visceral fat area, LDL.C: low-density lipoprotein cholesterol, TG: triglyceride, HDL.C: high-density lipoprotein cholesterol, PG: plasma glucose, OGTT: oral glucose tolerance test, Cr: serum creatinine, eGFR: estimated glomerular filtration rate, PWV: pulse wave velocity, ABI: anklebrachial index, IMT: intima media thickness

\section{Health guidance ("Hokenshido")}

After the health checkup, all of the participants receive the results of the health checkup and "Where am I?" chart (Fig. 1). To enhance understanding, all of the subjects were informed and given the opportunity to attend lectures by public health nurses and medical doctors.

In "Where am I?" chart, the metabolic risk factors of vascular damage were displayed at the bottom part of the chart. The results of detailed examination to estimate the current condition of vascular damage were put at the middle (of the chart). The status of presence or absence of diseases such as CVD was displayed at the top (of the chart). The placement of visceral fat accumulation at the bottom (part) implicates that metabolic risk factors, such as dyslipidemia, hyperglycemia, hypertension, and hyperuricemia are "the tip of the iceberg", and that visceral fat accumulation (dysregulation of adipocytokines in abdominal and visceral obesity) should induce the development of the risk factors, leading to atherosclerotic CVD and chronic kidney disease. Through this chart stream, the subjects having visceral fat accumulation can imagine their assumable stage for vascular damage, and be encouraged to alter their problematic lifestyle toward reducing visceral fat and cardiovascular risks. Such subjects are spontaneously helped to identify themselves as high risk subjects using this chart, based on the presence of multiple risk factors with visceral fat accumulation. On these conditions, health education "Hokenshido" program was provided by group and/or individual counseling. Public health nurse and dietitian interviewed and counseled the subjects about their pattern of meal, intake of alcohol, and habit of exercise. Through these processes, the guided subjects could determine the problematic habits which should be altered.

The number of subjects who received individual "Hokenshido" were $429(13.5 \%)$ in year 2003, and 123 (3.9\%) in year 2004. In particular, the subjects who could not improve their habit in the initial term were encouraged to repeatedly receive the group and individual lecture. The subjects considered already at high risk for CVD and chronic kidney diseases were referred to consult a cardiologist, neurologist, or nephrologist. Such subjects were continuously on the program to enhance the alteration of their problematic habits. 


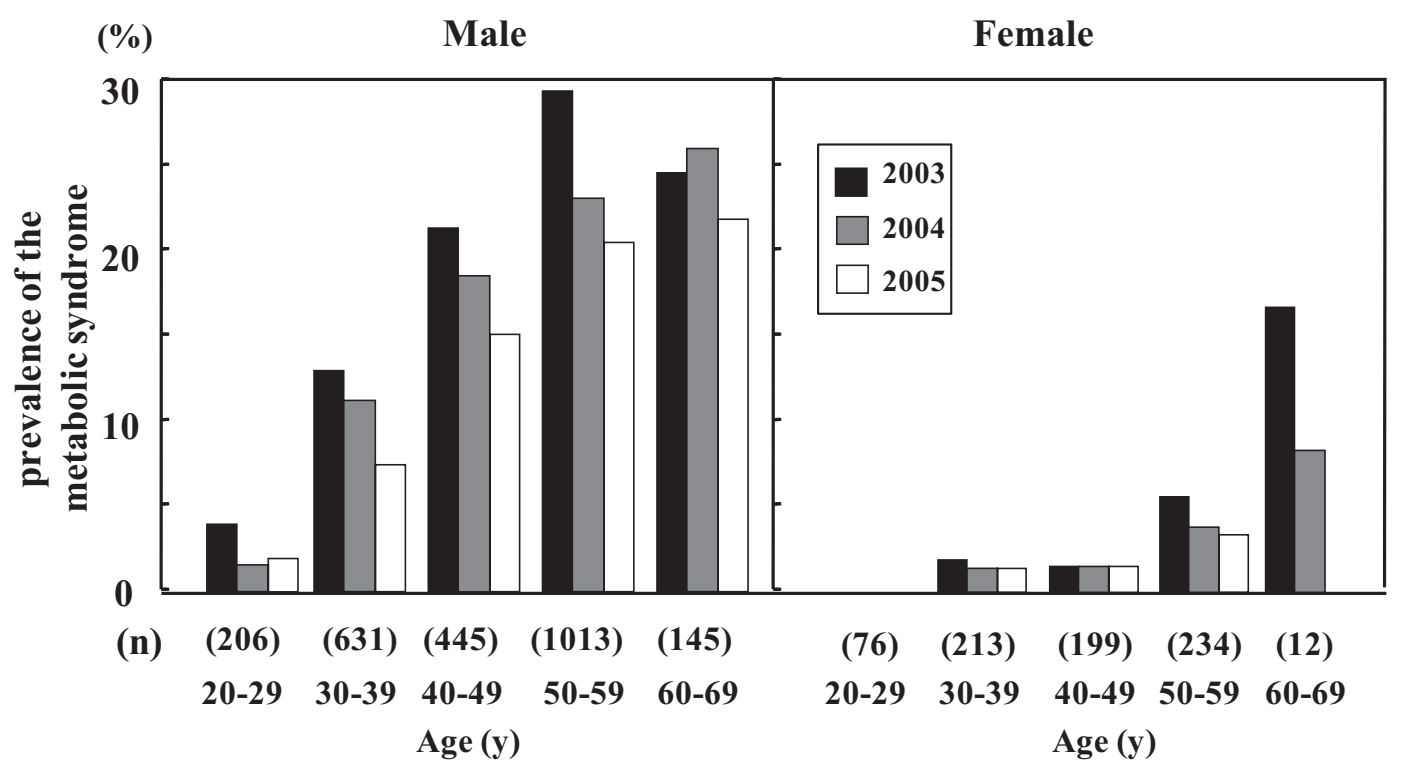

Figure 2. Age-related prevalence of the metabolic syndrome from year 2003 to year 2005. Male $(n=2,440)$, female $(n=734)$. Kruskal Wallis test with a Scheffe's test.

Table 2. Change in Waist Circumferences

\begin{tabular}{lcrllll}
\hline & \multirow{2}{*}{$\mathrm{n}$} & \multicolumn{3}{c}{ Waist circumference, cm } & \multirow{2}{*}{$\mathrm{p}$} \\
\cline { 4 - 6 } & & & year 2003 & year 2004 & year 2005 & \\
\hline Male & All & 2440 & $84.9 \pm 8.3$ & $84.1 \pm 8.4$ & $83.3 \pm 8.3$ & $<0.0001$ \\
& '03MS (+) & 508 & $92.8 \pm 6.7$ & $91.5 \pm 7.6$ & $90.3 \pm 7.4$ & $<0.0001$ \\
& '03MS (-) & 1932 & $82.8 \pm 7.4$ & $82.1 \pm 7.5$ & $81.5 \pm 7.5$ & $<0.0001$ \\
\hline Female & All & 734 & $78.2 \pm 10.0$ & $76.2 \pm 9.4$ & $76.7 \pm 9.8$ & $<0.001$ \\
& '03MS (+) & 22 & $98.3 \pm 5.5$ & $93.5 \pm 6.6$ & $94.4 \pm 6.8$ & $<0.05$ \\
& '03MS (-) & 712 & $77.6 \pm 9.4$ & $75.7 \pm 9.0$ & $76.2 \pm 9.3$ & $<0.001$ \\
\hline
\end{tabular}

Data are means \pm SD.

'03MS (+), with the metabolic syndrome at baseline; '03MS (-), without the metabolic syndrome at baseline.

Kruskal-Wallist test with a Scheffe's test

\section{Stastical analysis}

The comparison of prevalence of the metabolic syndrome and risk factors in the 3 year period were analyzed by Kruskal Wallis test with a Scheffe's test. The statistical significance of the differences in the waist circumferences in 3 years were also analyzed by Kruskal Wallis test with a Scheffe's test. All statistical analyses were performed with StatView-J 5.0 (SAS Inc.).

\section{Results}

Age-related prevalence of the metabolic syndrome increased from the age of 30 years and was the highest in the 50-59 year age group in males and increased after the age of 50 years in females (Fig. 2). After initiating measurement of waist circumference in annual health checkup, use of "Where am I?" chart, and "Hokenshido", the prevalence decreased among males aged 30-39 years, 40-49 years, and $50-59$ years $(\mathrm{p}<0.01)$, and among females aged 50-59 years, and 60-69 years during the 3-year period of this study.

The prevalence of the metabolic syndrome in 2003, 2004 and 2005 decreased in males $(20.8 \%, 17.2 \%, 14.4 \%, \mathrm{p}<$ $0.001)$ and females $(3.0 \%, 2.2 \%, 1.9 \%, \mathrm{p}=0.359)$. Decreased prevalence of the metabolic syndrome in males was associated with significant reductions in the prevalence of abdominal obesity, dyslipidemia, and hypertension $(\mathrm{p}<0.0001)$. Among subjects with metabolic syndrome at baseline, the number of subjects with metabolic syndrome significantly decreased in males $(508,287,247, \mathrm{p}<0.0001)$ and females $(22,8,6, \mathrm{p}<0.0001)$, respectively.

Significant reductions of waist circumference were seen in males and females (Table 2). Mean waist loss was $1.6 \mathrm{~cm}$ in males $(\mathrm{p}<0.0001)$ and $1.5 \mathrm{~cm}$ in females $(\mathrm{p}<0.001)$. Among subjects with metabolic syndrome at baseline, the mean waist loss was $2.5 \mathrm{~cm}$ in males $(\mathrm{p}<0.0001)$ and $3.9 \mathrm{~cm}$ in females $(\mathrm{p}<0.05)$.

To be noted, during the 3-year period of this study, no fatal atherosclerotic vascular events were recorded.

\section{Discussion}

In the present study, we demonstrated that 1) after initiating measurement of waist circumference in annual health 
checkup, use of "Where am I?" chart, and "Hokenshido" the prevalence of metabolic syndrome decreased with reductions in risk factors in males and females, 2) significant reductions of waist circumference were seen in males and females, 3) especially among males and females with the metabolic syndrome at baseline, the respective prevalence decreased markedly, and 4) fatal atherosclerotic vascular events were not recorded during the 3-year study period.

Based on the National Nutrition Survey in Japan, the rate of male obesity has been increasing. In this sense, the national campaign to improve the health of all Japanese people, called Kenko (Health) 21st, has not been fully successful. In the current study and program for the city employees, measurement of waist circumference and understanding of "Where am I?" chart seemed to be quite helpful to perceive their health conditions and reconsider the problematic habit. Through "Hokenshido", the guided subjects recognized a problem in their own lifestyle and attempted to reduce visceral fat as a goal to maintain a healthy life. In our Amagasaki Visceral Fat Study (8-17), we reported that the decrease in visceral fat was correlated with the decrease in the number of metabolic risk factors in the general male population (8).

Regarding the lack of fatal CVD events during the threeyear study period, improvements of risk factors and possibly also the improvements of adipocytokine dysregulation such as hypoadiponectinemia might stabilize arterial plaque.

A limitation of this study is that majority of blood samples were nonfasting. To enhance annual health checkup for as many employees, such blood sampling policy, either fasting or nonfasting, is allowed by the employer in many work places in Japan. Data were evaluated according to the criteria described in Materials and Methods, dependent on individual fasting or non-fasting conditions. Furthermore subjects with one or two risks without obesity should be also followed-up closely. In this study, 180 (5.7\%), 446 (14.1\%), and $162(5.1 \%)$ individuals were under treatment for dyslipidemia, hypertension, or diabetes, respectively, in year 2005.

Collectively, regular health checkups and "Hokenshido" program, which is based on the concept that visceral fat accumulation causes metabolic syndrome, effectively reduced the prevalence of the metabolic syndrome and various risk factors, which might lead to the prevention of CVD.

The authors state that they have no Conflict of Interest (COI).

\section{Acknowledgement}

We gratefully acknowledge Sachiko Morita (Amagasaki City Office) for research and comment, and Dr.Tomohiro Onda and Mitsuhiro Katashima (Kao Corporation) for technical assistance and comment.

\section{References}

1. Alberti KG, Zimmet P, Shaw J; IDF Epidemiology Task Force Consensus Group. The metabolic syndrome: A new worldwide definition. Lancet 366: 1059-1062, 2005.
2. Fujioka S, Matsuzawa Y, Tokunaga K, Tarui S. Contribution of intra-abdominal fat accumulation to the impairment of glucose and lipid metabolism in human obesity. Metabolism 36: 54-59, 1987.

3. Kanai H, Matsuzawa Y, Kotani K, et al. Close correlation of intraabdominal fat accumulation to hypertension in obese women. Hypertension 16: 484-490, 1990.

4. Nakamura T, Tokunaga K, Shimomura I, et al. Contribution of visceral fat accumulation to the development of coronary artery disease in non-obese men. Atherosclerosis 107: 239-246, 1994.

5. Grundy SM, Cleeman JI, Merz CN, et al. Implication of recent clinical trials for the National Cholesterol Education Program Adult Treatment Panel III guidelines. Circulation 110: 227-239, 2004.

6. Despres JP. Lemieux I. Abdominal obesity and metabolic syndrome. Nature 444: 881-887, 2006.

7. Grundy SM, Cleeman JI, Daniels SR, et al. Diagnosis and management of the metabolic syndrome: An American Heart Association/National Heart, Lung, and Blood Institute Scientific Statement. Circulation 112: 2735-2752, 2005.

8. Okauchi Y, Nishizawa H, Funahashi T, et al. Reduction of visceral fat is associated with decrease in the number of metabolic risk factors in Japanese men. Diabetes Care 30: 2392-2394, 2007.

9. Tamba S, Nishizawa H, Funahashi T, et al. Relationship between the serum uric acid level, visceral fat accumulation and serum adiponectin concentration in Japanese men. Intern Med 47: 11751180, 2008.

10. Akita EF, Okita K, Okauchi Y, et al. Impaired early insulin secretion in Japanese type 2 diabetes with metabolic syndrome. Diabetes Res Clin Pract 79: 482-489, 2008.

11. Okauchi Y, Kishida K, Funahashi T, et al. Changes in serum adiponectin concentrations correlate with changes in BMI, waist circumference, and estimated visceral fat area in middle-aged general population. Diabetes Care 32: e122, 2009.

12. Kamada $Y$, Nakamura $T$, Funahashi $T$, et al. Visceral obesity and hypoadiponectinemia are significant determinants of hepatic dysfunction: An epidemiologic study of 3827 Japanese subjects. J Clin Gastroenterol 43: 995-1000, 2009.

13. Tamba S, Nakatsuji H, Kishida K, et al. Relationship between visceral fat accumulation and urinary albumin-creatinine ratio in middle-aged Japanese men. Atherosclerosis 211: 601-605, 2010.

14. Nakatsuji H, Kishida K, Funahashi T, et al. One-year reductions in body weight and blood pressure, but not in visceral fat accumulation and adiponectin, improve urinary albumin-to-creatinine ratio in middle-aged Japanese men. Diabetes Care 33: e110-e111, 2010.

15. Okauchi Y, Kishida K, Funahashi T, et al. Absolute value of bioelectrical impedance analysis-measured visceral fat area with obesity-related cardiovascular risk factors in Japanese workers. J Atheroscler Thromb 17: 1237-1245, 2010.

16. Okauchi Y, Kishida K, Funahashi T, et al. 4-year follow-up of cardiovascular events and changes in visceral fat accumulation after health promotion program in the Amagasaki Visceral Fat Study. Atherosclerosis 212: 698-700, 2010.

17. Akita EF, Iwahashi H, Okauchi Y, et al. Predictors of deterioration of glucose tolerance and effects of lifestyle intervention aimed at reducing visceral fat in normal glucose tolerance subjects with abdominal obesity. J Diabetes Invest 2: 218-224, 2011.

18. Tokunaga K, Matsuzawa Y, Ishikawa K, Tarui S. A novel technique for the determination of body fat by computed tomography. Int J Obes 7: 437-445, 1983.

19. Matsuzawa Y. Metabolic syndrome-definition and diagnostic criteria in Japan. J Atheroscler Thromb 12: 301, 2005.

20. Eberly LE, Stamler J, Neaton JD; Multiple Risk Factor Intervention Trial Research Group. Relation of triglyceride levels, fasting and nonfasting, to fatal and nonfatal coronary heart disease. Arch Intern Med 163: 1077-1083, 2003.

21. Ahmad J, Hameed B, Das G, Siddiqui M, Ahmad I. Postprandial 
Intern Med 50: 1643-1648, 2011 DOI: 10.2169/internalmedicine.50.5039

hypertriglyceridemia and carotid intima-media thickness in north Indian type 2 diabetic subjects. Diabetes Res Clin Pract 69: 142150, 2005.
22. American Diabetes Association. Clinical practice recommendations 2000: screening for type 2 diabetes. Diabetes Care 23: S20-S23, 2000 .

(C) 2011 The Japanese Society of Internal Medicine http://www.naika.or.jp/imindex.html 